Journal of Environmental
Analysis and Progress
ISSN: $2525-815 \mathrm{X}$

\title{
Avaliação do potencial eólico da região do Vale do Jaguaribe no semiárido cearense utilizando modelagem numérica regional
}

\section{Wind power assessment at the Jaguaribe's Valley in the Ceará State's semiarid using regional numerical modeling}

\author{
Daniel von Glehn dos Santos ${ }^{\mathrm{a}}$, Camila Cristina Souza Lira ${ }^{\mathrm{b}}$, João Bosco Verçosa Leal Junior ${ }^{\mathrm{a}}$ \\ a Universidade Estadual do Ceará-UECE, Campus do Itaperi, Avenida Silas Munguba, n. 1700, Itaperi, Fortaleza, Ceará, \\ Brasil. CEP: 60714-903. E-mail: danielvonglehn@gmail.com, jblealjr@gmail.com.

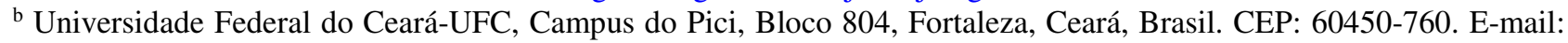 \\ eng.camilalira@gmail.com.
}

\begin{tabular}{|c|c|}
\hline A R T I C L E I N F O & A B S T R A C T \\
\hline Recebido 12 Dez 2017 & The Aracati wind is a well-known phenomenon in the Jaguaribe's river basin local \\
\hline Aceito 23 Jan 2018 & community. However, its wind power is still poorly studied and explored. It was the \\
\hline Publicado 23 Jan 2018 & primary justification for this study, that was to evaluate the wind power of the Aracati \\
\hline & wind affected area through regional numerical modeling with the WRF model. \\
\hline & $\begin{array}{l}\text { Comparisons are made between the simulation output and the observation data at } \\
\text { some cities nearby the Jaguaribe river to validate the models. The Weather Research }\end{array}$ \\
\hline & and Forecasting (WRF) model represented well the wind speed, with Willmott's \\
\hline & indexes up to 0.7709 in Jaguaruana. When it comes to wind power, according to the \\
\hline & $\begin{array}{l}\text { National Renewable Energy Laboratory (NREL) assessment criteria, Jaguaruana } \\
\text { showed the best results, considered Satisfactory, Morada Nova, as Marginal, and }\end{array}$ \\
\hline & Jaguaribe and Iguatu, as Poor. \\
\hline
\end{tabular}

Keywords: Wind power, WRF, Aracati wind.

\begin{abstract}
R E S U M O
O vento Aracati é um fenômeno bastante conhecido pelos moradores da região da bacia do Rio Jaguaribe. Entretanto, seu potencial eólico é pouco estudado e explorado. Esta é a principal justificativa deste estudo, o qual visou avaliar o potencial eólico da região cearense do Vale do Jaguaribe afetada pelo vento Aracati através de modelagem numérica regional com o modelo Weather Research and Forecasting (WRF). Comparações foram realizadas com os resultados da simulação com os dados observados em alguns municípios nas proximidades do rio Jaguaribe, como forma de validação do modelo. $\mathrm{O}$ modelo utilizado representou de forma satisfatória a velocidade do vento, com índices de Willmott de até 0,7709 em Jaguaruana. Quanto ao potencial eólico, de acordo com os critérios de classificação da National Renewable Energy Laboratory (NREL) dos EUA, o município de Jaguaruana apresentou o melhor resultado, considerado Satisfatório, Morada Nova, como Marginal, e Jaguaribe e Iguatu como Pobres.

Palavras-Chave: Energia eólica, WRF, vento Aracati.
\end{abstract}

\section{Introdução}

O vasto litoral e o clima do Brasil são conhecidos por favorecerem a geração de energia a partir do vento. Mas não só o litoral é propício à geração de energia elétrica pela fonte eólica, o semiárido cearense também pode ter um papel importante nesse contexto. Além disso, na região leste do estado, o vento Aracati também favorece a geração de energia, levando conforto térmico desde o litoral leste até alguns municípios do sertão sul do Estado. "A explicação para o surgimento do Aracati em regiões mais distantes do litoral pode estar associada ao Rio Jaguaribe, o qual desempenharia um papel importantíssimo no sentido de servir como canalizador do vento" (Camelo \& Leal Junior, 2015).

A implantação de turbinas ou parques eólicos, em qualquer que seja o local, exige um 
estudo prévio da área, a fim de designar o equipamento mais adequado às condições do local. Porém, muitas vezes, tem-se a dificuldade de encontrar dados confiáveis para o estudo de viabilização do parque eólico. Para isso, os modelos numéricos têm o importante papel de suprir a ausência desses dados e, mais além, de prever possíveis cenários futuros.

Os modelos numéricos atmosféricos resolvem numericamente as equações do escoamento atmosférico e equações parametrizadas de fenômenos de pequena escala. $\mathrm{O}$ modelo utilizado neste estudo, o Weather Research and Forecasting (WRF) é definido no manual técnico, por Skamarock et al. (2008), como um sistema de previsão numérica do tempo e de simulação atmosférica, desenvolvido tanto para pesquisa quanto para aplicações práticas.

Camelo (2007) realizou um dos primeiros estudos acerca da ocorrência do Aracati, através de um estudo numérico e de análise de dados observados. Outro estudo importante a ser citado é o de Vasconcelos Júnior (2010), que investigou a relação do vento Aracati com os Jatos de Baixos Níveis, também através de modelagem numérica. Estudos como esses, revelam a importância e utilidade das ferramentas de modelagem numérica.

Dentro desse escopo, este estudo tem como objetivo avaliar o potencial eólico da região cearense do Vale do Jaguaribe, que recebe influência do vento Aracati, utilizando modelagem numérica regional com o modelo WRF.

\section{Material e Métodos}

Local de estudo

O local escolhido para a realização deste estudo é a região onde o vento Aracati é observado, que, de acordo com Camelo (2007), se dá nas proximidades do Rio Jaguaribe e seus afluentes, desde o litoral leste até as proximidades das cidades de Icó e Iguatu, ao sul do Estado. A região de estudo é mostrada na Figura 1.

Gatto (1999) caracteriza a bacia hidrográfica do rio Jaguaribe, como estando situada em sua quase totalidade dentro dos limites do Estado do Ceará e ocupando cerca de 51,9\% da área total do Estado. O rio Jaguaribe e seus afluentes percorrem uma região dominada, basicamente, pela Caatinga, Savana-Estepe e Estepe-Floresta e $60 \%$ da bacia é abrangida pelo clima semiárido, que ocorre no sentido NE-SO, do litoral até o extremo sudoeste.

Localizado na zona intertropical, próximo da linha do equador, o clima da região cearense pode ser separado em dois períodos do ano: as estações chuvosa e seca. A estação das chuvas mais significativas inicia-se em dezembro e se estende até junho ou julho, dependendo das condições atmosféricas e oceânicas atuantes. Em fevereiro de cada ano inicia-se a chamada quadra chuvosa do Estado do Ceará, que se estende até maio.

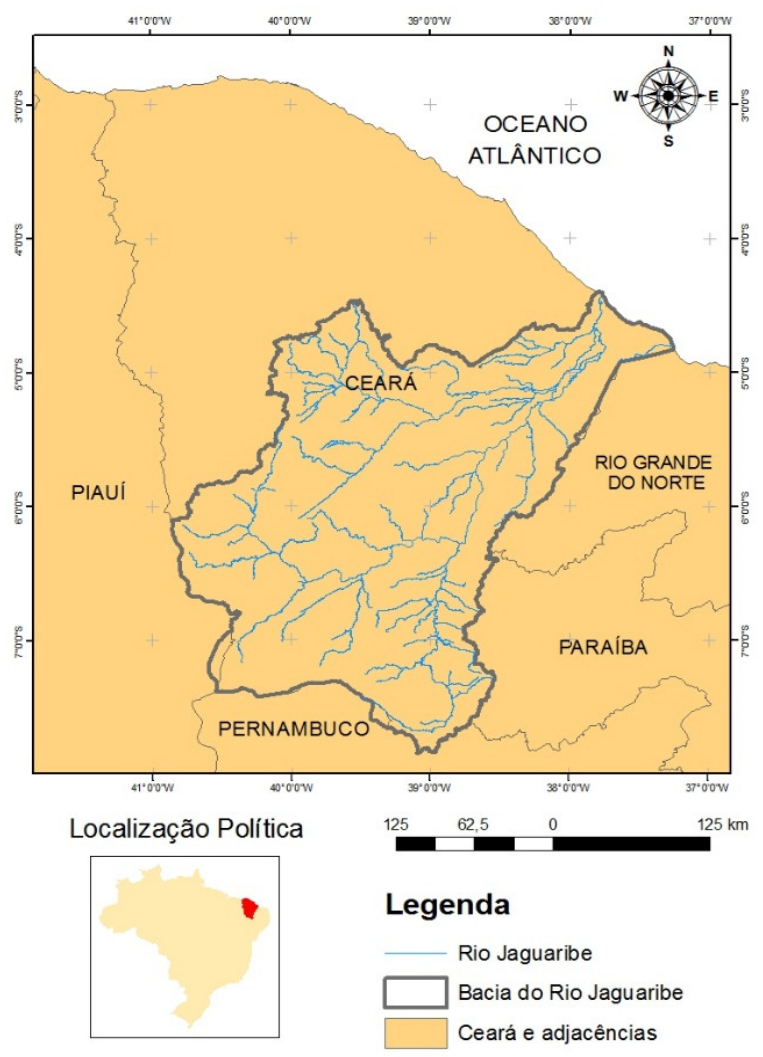

Figura 1. Área de estudo na Bacia do Rio Jaguaribe, Ceará, Brasil. 


\section{Período de estudo}

Foi realizada uma simulação no período seco, na primeira semana de outubro de 2015, iniciada às $18 \mathrm{~h} 00 \mathrm{~min}$ UTC $(15 \mathrm{~h} 00 \mathrm{~min}$ local $)$ do dia 01/10/2015 e encerrada às 00:00 UTC $(21 \mathrm{~h} 00 \mathrm{~min}$ local) do dia 09/10/2015. As primeiras seis horas foram descartadas da análise como tempo de ajuste do modelo, ou spin up.

\section{Simulação}

Os dados de entrada para as condições iniciais e de contorno para as simulações no WRF são os dados de 6 horas do CFSv2, com resolução de $0,5^{\circ} \times 0,5^{\circ}$ para os níveis de pressão e de $0,205^{\circ}$ $\mathrm{x} \sim 0,204^{\circ}$ aos níveis da superfície e abaixo do solo.
O modelo foi inicializado com três domínios aninhados: d01 (151 x 121 pontos de grade com 16,2 km de resolução), d02 (121 x 121 pontos de grade com $5,4 \mathrm{~km}$ de resolução) e d03 (151 x 151 pontos de grade com $1,8 \mathrm{~km}$ de resolução); todos centralizados na latitude $5,25^{\circ} \mathrm{S}$ e longitude $38,4^{\circ} \mathrm{W}$, com projeção cartográfica de Mercator. O passo de tempo é adaptativo e a resolução vertical é de 45 pontos de grade, com pressão de $100 \mathrm{hPa}$ ao topo e utilizando uma distribuição hiperbólica tangente, com maior densidade de níveis nas proximidades da base e do topo do domínio.

A Figura 2 ilustra a localização, distribuição e aninhamento dos três domínios utilizados na simulação.

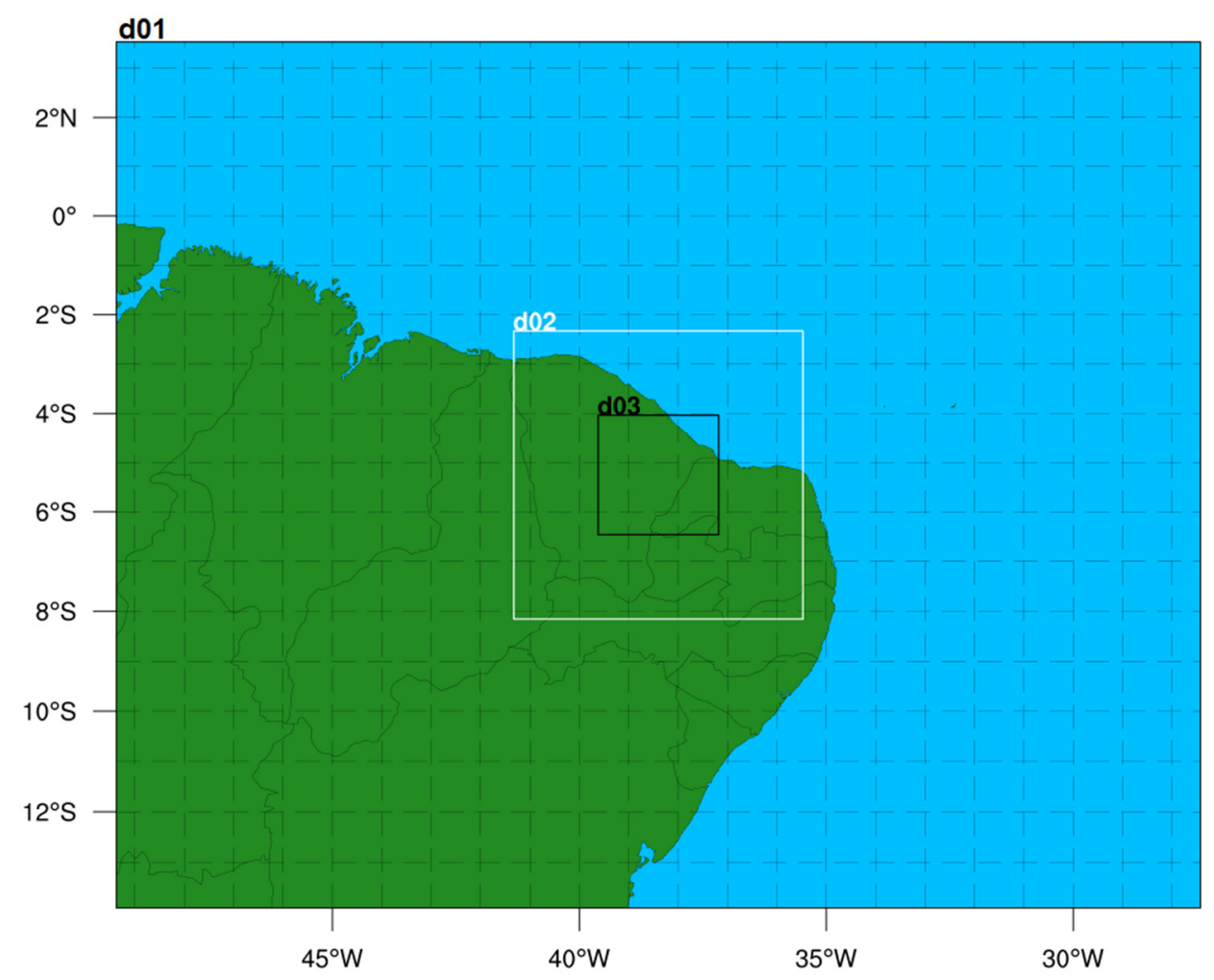

Figura 2. Domínios da simulação com o modelo Weather Research and Forecasting (WRF).

As parametrizações utilizadas na simulação com o modelo WRF foram escolhidas baseando-se nos estudos de Silva (2013) e Silva \& Fisch (2014) e são elas:

a) Microfísica: WRF Single-moment 6-class Scheme (Hong \& Lim, 2006);

b) Parametrização de Cumulus: Kain-Fritsch Scheme (Kain, 2004);

c) Modelo de Superfície: Unified Noah Land Surface Model (Tewari et al., 2004);

d) Camada Limite Planetária: Mellor-Yamada Nakanishi Niino (MYNN) Level 2.5 Scheme (Nakanishi e Niino, 2006);

e) Camada de superfície: MYNN Scheme (Nakanishi \& Niino, 2006); f) Radiação de onda curta: Dudhia Shortwave Scheme (Dudhia, 1989);

g) Radiação de onda longa: RRTM Longwave Scheme (Mlawer et al., 1997).

\section{Comparação com dados observados}

Para a verificação dos resultados da simulação, utilizou-se de dados de velocidade do vento médios horários medidos a $10 \mathrm{~m}$ de altura pelas Plataformas de Coletas de Dados (PCD) do Instituto Nacional de Meteorologia (INMET), nos municípios de Morada Nova $\left(5,136^{\circ} \mathrm{S} ; 38,356^{\circ}\right.$ $\mathrm{W})$, Jaguaruana $\left(4,787^{\circ} \mathrm{S} ; 37,777^{\circ} \mathrm{W}\right)$, Jaguaribe 
$\left(5,905^{\circ} \mathrm{S} ; 38,628^{\circ} \mathrm{W}\right)$ e Iguatu $\left(6,397^{\circ} \mathrm{S} ; 39,270^{\circ}\right.$ $\mathrm{W})$.

Na comparação da previsão com os dados observados, como critério quantitativo, utilizou-se o Erro Absoluto Médio (MAE), a Raiz do Erro Quadrático Médio (RMSE) e o índice de Willmott (Willmott, 1981):

$$
\begin{gathered}
M A E=\frac{1}{n} \sum_{i=1}^{n}\left|S_{i}-O_{i}\right| \\
R M S E=\sqrt{\frac{1}{n} \sum_{i=1}^{n}\left(S_{i}-O_{i}\right)^{2}} \\
d=1-\frac{\sum_{i=1}^{n}\left(S_{i}-O_{i}\right)^{2}}{\sum_{i=1}^{n}\left[\left|S_{i}-\bar{O}\right|+\left|O_{i}-\bar{O}\right|\right]^{2}}
\end{gathered}
$$

onde: $d=$ índice de Willmott; $S_{i}=$ valor simulado de ordem $i ; O_{i}=$ valor observado de ordem $i ; \bar{O}=$ média dos valores observados; e $n=$ número de eventos.

\section{Avaliação do potencial eólico}

Para avaliação do potencial eólico, utilizou-se o critério do Laboratório Nacional de Energia Renovável (NREL, do inglês National Renewable Energy Laboratory) dos EUA, que determina a qualidade do potencial eólico de uma determinada região associando classes de valores de densidade de potência eólica $\left(\mathrm{D}_{\mathrm{Pm}}\right)$ às classes de velocidade média do vento $\left(\mathrm{v}_{\mathrm{m}}\right)$ a $10 \mathrm{~m}$ e a $50 \mathrm{~m}$. O Quadro 1 mostra a classificação do NREL.

Quadro 1. Classificação do potencial eólico para a velocidade média do vento $\left(\mathrm{m} \cdot \mathrm{s}^{-1}\right)$ e para a Densidade de Potência Média (W.m ${ }^{-2}$ ), a $10 \mathrm{~m}$ e a $50 \mathrm{~m}$ da superfície (NREL, 2016). Fonte: NREL (2016).

\begin{tabular}{|c|c|c|c|c|c|}
\hline Classe de vento & $\left.\mathbf{D}_{\mathbf{P m}} \mathbf{( 1 0} \mathbf{~ m}\right)$ & $\left.\mathbf{v}_{\mathbf{m}} \mathbf{( 1 0} \mathbf{~ m}\right)$ & $\left.\mathbf{D}_{\mathbf{P m}} \mathbf{( 5 0} \mathbf{~ m}\right)$ & $\left.\mathbf{v}_{\mathbf{m}} \mathbf{( 5 0} \mathbf{~ m}\right)$ & Descrição \\
\hline 1 & 0 a 100 & 0 a 4,4 & 0 a 200 & 0 a 5,6 & Pobre \\
\hline 2 & 100 a 150 & 4,4 a 5,1 & 200 a 300 & 5,6 a 6,4 & Marginal \\
\hline 3 & 150 a 200 & 5,1 a 5,6 & 300 a 400 & 6,4 a 7,0 & Satisfatório \\
\hline 4 & 200 a 250 & 5,6 a 6,0 & 400 a 500 & 7,0 a 7,5 & Bom \\
\hline 6 & 250 a 300 & 6,0 a 6,4 & 500 a 600 & 7,5 a 8,0 & Excelente \\
\hline 7 & 300 a 350 & 6,4 a 7.0 & 600 a 800 & 8,0 a 8,8 & Proeminente \\
\hline & 350 a 400 & 7,0 a 9,4 & 800 a 2000 & 8,8 a 11,9 & Esplêndido \\
\hline
\end{tabular}

\section{Resultados}

Após realizada a simulação, as seis primeiras horas da simulação foram removidas, e o período aproveitado da simulação foi de $168 \mathrm{~h}$ (uma semana), compreendidos entre às $21 \mathrm{~h} 00 \mathrm{~min}$ de 01 de outubro de 2015 e às $21 \mathrm{~h} 00 \mathrm{~min}$ de 08 de outubro de 2015, horário local. Os campos de velocidade média do vento na semana simulada estão ilustrados na Figura 3.

A avaliação dos resultados da simulação baseou-se apenas nos dados de velocidade do vento a $10 \mathrm{~m}$, e se deu através de comparações entre o modelo WRF e os dados das PCD. A comparação realizada de forma quantitativa, através dos métodos estatísticos descritos na metodologia, é mostrada na Tabela 1.
Tabela 1. Medidas estatísticas entre os dados do Weather Research and Forecasting (WRF) e das Plataformas de Coletas de Dados (PCD).

\begin{tabular}{lccc}
\hline Município & $\begin{array}{c}\text { MAE } \\
\left(\mathbf{m . s}^{-1}\right)\end{array}$ & $\begin{array}{c}\text { RMSE } \\
\left(\mathbf{m . s}^{-\mathbf{1}}\right)\end{array}$ & $\boldsymbol{d}$ \\
\hline Jaguaruana & 1,156 & 1,426 & 0,7709 \\
Morada & 2,051 & 2.417 & 0,6244 \\
Nova & & 1,986 & 0,6689 \\
Jaguaribe & 1,608 & 1,776 & 0,599 \\
\hline Iguatu & 1,422 & 1,
\end{tabular}

Por fim, utilizando os critérios do NREL apresentado no Quadro 1, foi feita a classificação do potencial eólico baseado nos resultados da simulação a $10 \mathrm{~m}$ e $50 \mathrm{~m}$, em cada um dos municípios estudados. O resultado da classificação é mostrado na Tabela 2, juntamente com os valores de velocidade média $\left(\mathrm{em} \mathrm{m} . \mathrm{s}^{-1}\right)$ da semana estudada. 


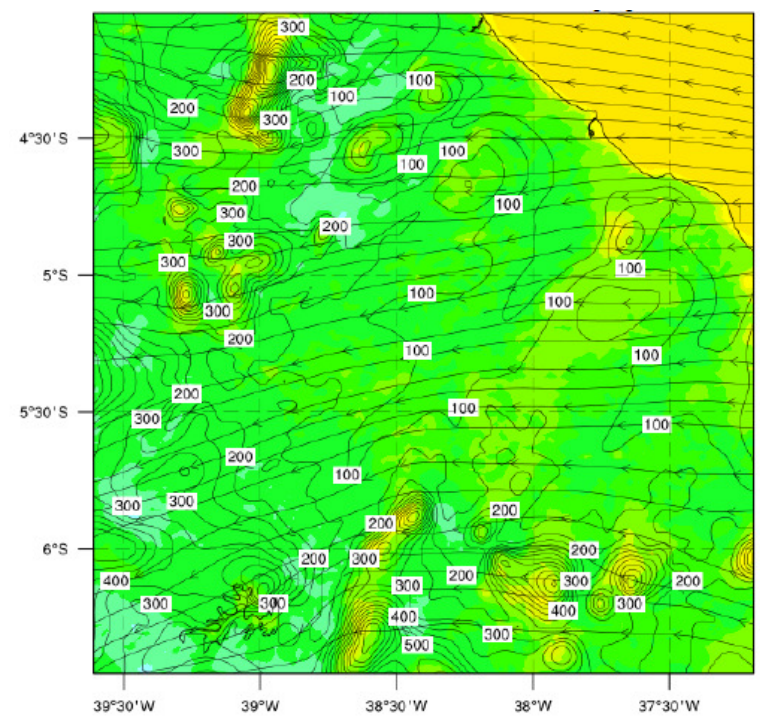

(a)

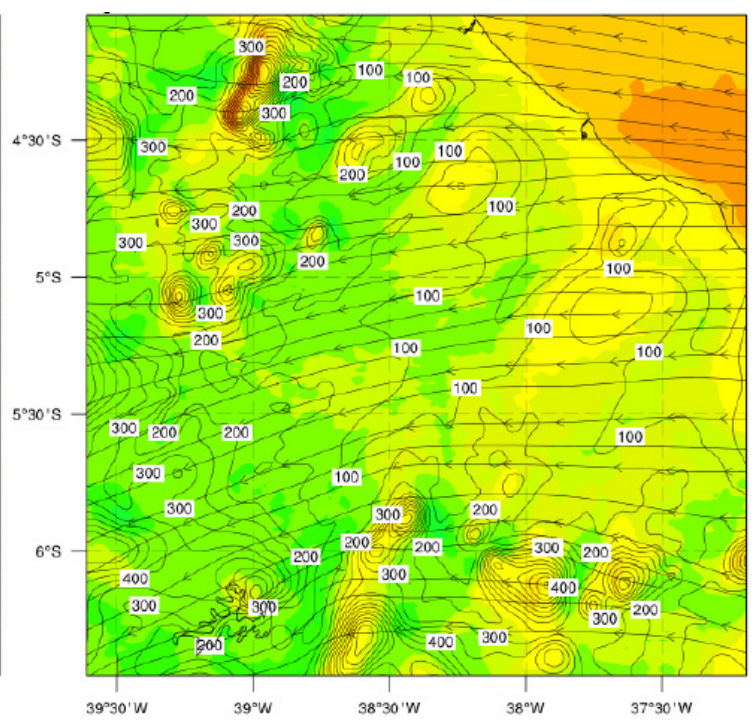

(b)

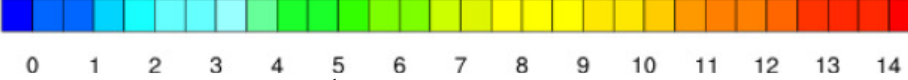

Figura 3. Campo de velocidade do vento $\left(\mathrm{m}_{\mathrm{s}} \mathrm{s}^{-1}\right)$ e linhas de corrente a (a) $10 \mathrm{~m}$ e a (b) $50 \mathrm{~m}$.

Tabela 2. Classificação do potencial eólico segundo os critérios do Laboratório Nacional de Energia Renovável (NREL, do inglês National Renewable Energy Laboratory) para os municípios estudados e para as velocidades médias $(\mathrm{m} / \mathrm{s})$ a $10 \mathrm{~m}$ e a $50 \mathrm{~m}$ da superfície.

\begin{tabular}{ccccccc}
\hline Município & $\mathbf{1 0 ~}$ & \multicolumn{2}{c}{ Classe e Descrição } & \multicolumn{5}{c}{$\mathbf{5 0 ~ m}$} & \multicolumn{2}{c}{ Classe e Descrição } \\
\hline Jaguaruana & 5.4381 & 3 & Satisfatório & 6.8439 & 3 & Satisfatório \\
Morada Nova & 4.6964 & 2 & Marginal & 5.9104 & 2 & Marginal \\
Jaguaribe & 4.3497 & 1 & Pobre & 5.4741 & 1 & Pobre \\
Iguatu & 3.8303 & 1 & Pobre & 4.8204 & 1 & Pobre \\
\hline
\end{tabular}

\section{Discussão}

É possível observar na Figura 3 a predominância do vento leste, efeito dos ventos alísios. Verifica-se também, em ambas as alturas, maiores valores de velocidade do vento na região do Vale do Jaguaribe em relação a seus arredores, devido à canalização do vento, que é acelerado vale adentro, na forma de um vento catabático.

Ao avaliar a Tabela 1, pode-se notar pelos erros que, em termos de velocidade do vento, o modelo WRF se saiu melhor em Jaguaruana, com MAE $=1,156 ;$ RMSE $=1,426$ e Willmott $=0,7709$.

$\mathrm{Na}$ Tabela 2, os municípios estão organizados em ordem crescente em relação a sua distância do litoral, dessa forma nota-se que a qualidade do potencial eólico decresce com a distância do litoral. Tal comportamento é esperado devido às perdas de energia do escoamento pelo atrito e cisalhamento. O município de Jaguaruana apresentou melhor potencial eólico alcançando classificação satisfatória. Apesar de ter sido classificado como pobre, o vento de Jaguaribe esteve próximo dos limiares entre pobre e marginal, tanto a $10 \mathrm{~m}$ como a $50 \mathrm{~m}$.

\section{Conclusão}

A modelagem numérica se mostrou uma ferramenta importante para este e outros estudos de potencial eólico e, apesar dos erros encontrados, resultados relevantes foram obtidos, embora ainda seja interessante a realização de mais testes para o caso. O modelo WRF apresenta uma gama vasta de parametrizações e configurações que merecem ser testadas a fim de se alcançar os melhores resultados possíveis.

Futuros estudos a respeito desse caso estão em andamento, como a realização das simulações alterando-se a base de dados na inicialização e condições de contorno, as dimensões das grades, a escolha das parametrizações e executando simulações em outros períodos do ano.

Esse estudo pode ser de grande importância para pesquisadores da área de modelagem numérica regional e energia eólica, como uma referência de teste e aplicação ou até mesmo como motivação para futuras pesquisas no semiárido brasileiro.

\section{Agradecimentos}

Os autores agradecem ao Laboratório LIMMA, ao Curso de Mestrado Acadêmico em Ciências Físicas Aplicadas, à Universidade 
Estadual do Ceará (UECE) e ao Instituto Nacional de Meteorologia do Brasil (INMET), pela disponibilização dos dados observados.

\section{Referências}

CAMELO, H. N. 2007. Estudo Numérico do Vento Aracati para Caracterização de seu Potencial Eólico. Mai. 98f. Dissertação (Mestrado Acadêmico em Ciências Físicas Aplicadas), Centro de Ciências e Tecnologia, Universidade Estadual do Ceará, Fortaleza.

CAMELO, H. N.; LEAL JUNIOR, J. B. V. 2015. Estudo Numérico do Vento Aracati, um Evento Frequente de Rio Perene no Estado do CearáBrasil. Revista Brasileira de Geografia Física, v. 8, n. 2, p. 334-351.

GATTO, L. C. S. 1999. Diagnóstico Ambiental da Bacia do Rio Jaguaribe. IBGE, Salvador. Disponível em: ftp://geoftp.ibge.gov.br/documentos/recursos_natu rais/diagnosticos/jaguaribe.pdf. Acesso em: 04 mai. 2016.

NREL. Wind Energy Resource Atlas of the United States. 2016. Disponível em: http://rredc.nrel.gov/wind/pubs/atlas. Acesso em: 19 set.

SKAMAROCK, W. C.; KLEMP, J. B.; DUDHIA, J.; GILL, D. O.; BARKER, D. M.; DUDA, M. G.; HUANG, X.; WANG, W.; POWERS, J. G. NCAR 2008. Technical Note: A Description of the Advanced Research WRF Version 3 (NCAR/TN475+STR). Boulder, Colorado, USA: Nacional Center for Atmospheric Research.

SILVA, A. F. G. 2013. Avaliação do Modelo WRF ao Perfil do Vento no Centro de Lançamento de Alcântara. 98p. (sid.inpe.br/mtcm19/2013/02.07.16.21-TDI). Dissertação (Mestrado em Meteorologia), Instituto Nacional de Pesquisas Espaciais (INPE), São José dos Campos.

SILVA, A. F. G.; FISCH, G. 2014. Avaliação do Modelo WRF para a Previsão do Perfil do Vento no Centro de Lançamento de Alcântara. Revista Brasileira de Meteorologia, v. 29, n. 2, p. 259-270.

VASCONCELOS JÚNIOR, F. DAS C. 2010. Estudo Numérico de Jatos de Baixos Níveis do Estado do Ceará. Fev. 130f. Dissertação (Mestrado Acadêmico em Ciências Físicas Aplicadas), Centro de Ciências e Tecnologia, Universidade Estadual do Ceará, Fortaleza.

WILLMOTT, C. J. 1981. On the validation of models. Physical Geography, v. 2, n. 2, p. 184-194.

HONG, S.-Y.; LIM, J.-O. J. 2006. The WRF single-moment 6-class microphysics scheme (WSM6). Journal of Korean Meteorology Society, v. 42 , p. 129-151.

KAIN, J. S. 2004. The Kain-Fritsch convective parameterization: An update. Journal of Applied Meteorology and Climatology, v. 43, p. 170-181.

TEWARI, M.; CHEN, F.; WANG, W.; DUDHIA, J.; LEMONE, M. A.; MITCHELL, K.; EK, M.; GAYNO, G.; WEGIEL, J.; CUENCA, R. H. 2004. Implementation and verification of the unified NOAH land surface model in the WRF model. 20th conference on weather analysis and forecasting/16th conference on numerical weather prediction, pp. 11-15.

NAKANISHI, M., NIINO, H. 2006. An improved Mellor-Yamada level 3 model: its numerical stability and application to a regional prediction of advecting fog. Boundary-Layer Meteorology, v. 119 , p. 397-407.

DUDHIA, J. 1989. Numerical study of convection observed during the Winter Monsoon Experiment using a mesoscale two-dimensional model. Journal of the Atmospheric Sciences, v. 46, p. 3077-3107.

MLAWER, E. J.; TAUBMAN, S. J.; BROWN, P. D.; IACONO, M. J.; CLOUGH, S. A. 1997. Radiative transfer for inhomogeneous atmospheres: RRTM, a validated correlated-k model for the longwave. Journal of Geophysical Research, v. 102, p. 16663-16682. 